ARTICLE

Received 4 Jul 2014 | Accepted 10 Oct 2014 | Published 26 Nov $2014 \quad$ DOl: 10.1038/ncomms6544

\title{
Cytoplasmic dynein transports cargos via load-sharing between the heads
}

\author{
Vladislav Belyy ${ }^{1}$, Nathan L. Hendel ${ }^{2}$, Alexander Chien $^{1} \&$ Ahmet Yildiz 2,3
}

Cytoplasmic dynein is a motor protein that walks along microtubules (MTs) and performs mechanical work to power a variety of cellular processes. It remains unclear how a dynein dimer is able to transport cargos against load without coordinating the stepping cycles of its two heads. Here by using a DNA-tethered optical trapping geometry, we find that the forcegenerating step of a head occurs in the MT-bound state, while the 'primed' unbound state is highly diffusional and only weakly biased to step towards the MT-minus end. The stall forces of the individual heads are additive, with both heads contributing equally to the maximal force production of the dimer. On the basis of these results, we propose that the heads of dynein utilize a 'load-sharing' mechanism, unlike kinesin and myosin. This mechanism may allow dynein to work against hindering forces larger than the maximal force produced by a single head.

\footnotetext{
${ }^{1}$ Biophysics Graduate Group, University of California at Berkeley, Berkeley, California 94720, USA. ${ }^{2}$ Department of Physics, University of California at Berkeley, Berkeley, California 94720, USA. ${ }^{3}$ Department of Cellular and Molecular Biology, University of California at Berkeley, Berkeley, California 94720, USA. Correspondence and requests for materials should be addressed to A.Y. (email: yildiz@berkeley.edu).
} 
C ytoplasmic dynein (herein referred to as dynein) motors walk processively towards the microtubule (MT)-minus end and generate forces of several $\mathrm{pN}^{1,2}$. The mechanical work produced by dynein motors has a broad range of cellular functions, including cargo transport, mitotic spindle positioning and organization of the MT network ${ }^{3}$. Despite dynein's central roles in neurobiology and development, the mechanism of its force production remains poorly understood in comparison with other molecular motors, in part because of its large size and complex structure ${ }^{4}$.

Dynein is a homodimer of two $\sim 500-\mathrm{kDa}$ heavy chains. In contrast to kinesin and myosin, which have a single ATP-binding site per motor domain, the dynein motor domain (head) contains six AAA + ATPase subunits arranged into a hexameric ring (Fig. 1a). Four of the AAA + subunits bind nucleotide and the AAA1 subunit serves as the primary site of ATP hydrolysis. The $\mathrm{AAA}+$ ring connects to a MT via a $15-\mathrm{nm}$ coiled-coil stalk bearing a small MT-binding domain (MTBD), resulting in a $\sim 25-\mathrm{nm}$ separation between the MTBD and the AAA1 site ${ }^{5,6}$. The two rings dimerize through an N-terminal tail domain, which also serves as the binding site for a number of light chains and adapter proteins ${ }^{7}$. Dynein-driven transport requires other

a

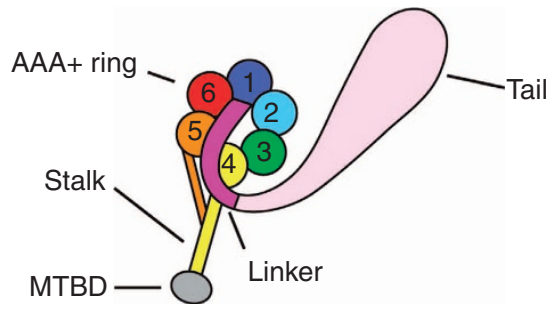

b
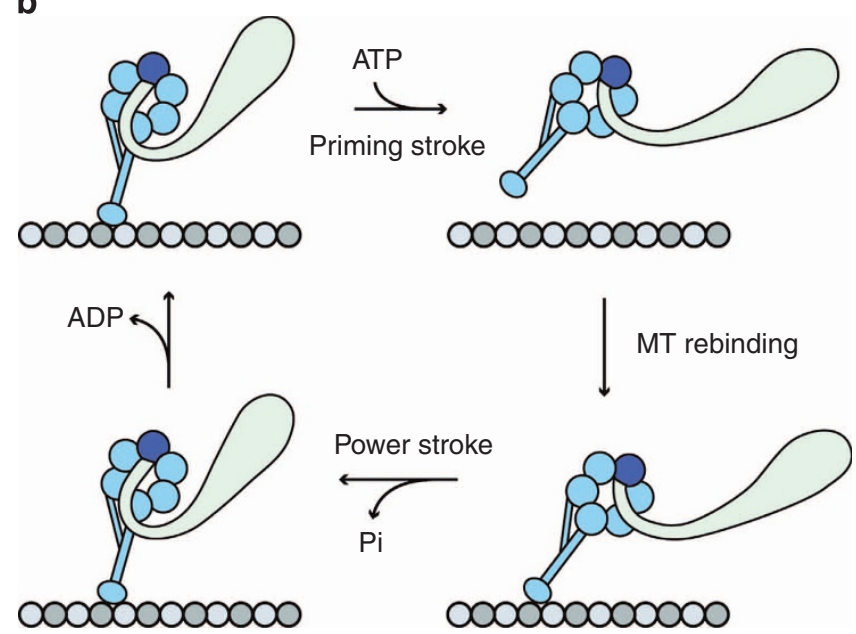

Figure 1 | Domain organization and mechanochemical cycle of

cytoplasmic dynein. (a) The dynein heavy chain consists of an N-terminal cargo-binding tail domain, an AAA + ATPase ring attached to the tail via the mechanically active linker and a MTBD separated from the AAA + ring by a $\sim 15-n m$ coiled-coil stalk. Individual AAA subunits are numbered 1 through 6. (b) Following the binding of ATP at the principal ATPase site (AAA1, coloured dark blue), the dynein head releases from the MT and its linker undergoes a priming stroke. The primed linker exits the ring at AAA2 rather than AAA4, and a dynein monomer attains an extended conformation. After the head re-binds the MT, its linker undergoes a power stroke, returning to its initial conformation exiting the ring at AAA4. The AAA1 site then releases ADP, completing the mechanochemical cycle. components, such as the cofactor dynactin, and regulatory proteins Lis1 and $\mathrm{NudE}^{4}$.

The following model of dynein's mechanochemical cycle has been proposed to explain how a dynein monomer generates force. ATP binding to the AAA1 site ${ }^{8}$ triggers the head's release from the MT and drives a priming stroke of the linker ${ }^{9}$. The linker, a long hinged domain at the base of the tail ${ }^{10,11}$, undergoes largescale conformational changes across the face of the AAA + ring in an ATP-dependent manner ${ }^{9,12,13}$ (Fig. 1b). Notably, the linker exits the ring at the AAA4 site in the unprimed state and at the AAA2 site in the primed state. The priming stroke has been proposed to move the stalk and MTBD of the unbound head towards the minus end of the $\mathrm{MT}^{9}$. After ATP hydrolysis, the head re-binds to MT at a new location and releases inorganic phosphate $^{10}$. The linker then undergoes a power stroke, generating tension in the process and returning the monomer to its unprimed state ${ }^{13}$. While intramolecular tension has been proposed to play a significant role in dynein motility, the magnitude of this tension remains to be measured directly. The proposed model does not explain how much mechanical work is produced by conformational changes of the linker and how two heads function together in a dimer to walk against a hindering load.

In this study, we use a head-tethered optical trapping geometry $^{14}$ to directly observe the force production and stepping of individual heads of a walking dynein dimer. We find that each head relies on diffusion to travel to the next binding site on the MT following its priming stroke. Force is then produced by the power stroke after the head rebinds to the MT. The stall forces of the two heads are approximately additive despite the absence of coordination in their stepping, leading us to propose a load sharing model for dynein-driven cargo transport. These results reveal unique properties of dynein force generation in comparison with other cytoskeletal motors.

\section{Results}

Development of head tethered dynein geometry. Force production of cytoskeletal motors has been studied extensively by attaching an optically trapped bead to the dimerization domain. Such assays directly measure the step size and stall force of the motor's tail ${ }^{2}$, providing detailed information about the load dependence of each head's stepping kinetics under the assumption that the heads alternately take steps and swap the leading position. However, dynein's heads can adapt a wide variety of orientations and step independently of each other ${ }^{15,16}$. Therefore, not much can be learned about the force production and stepping of an individual head from these assays.

In order to characterize the behaviour of a dynein head under load, we linked an optically trapped bead to one of the heads of GST-Dyn $1_{331 \mathrm{kDa}}$, a tail-truncated Saccharomyces cerevisiae dynein dimerized with glutathione $S$-transferase (GST) (Supplementary Fig. 1). This construct takes steps of the same size and duration as full-length dynein ${ }^{17}$, stalls at $\sim 70 \%$ of fulllength dynein's stall force ${ }^{2}$, and is herein referred to as wild type (WT). Dynein was covalently linked to a DNA tether at its C terminus (Fig. 2a). The labelling efficiency of the DNA tether to dynein was adjusted (Fig. $2 \mathrm{~b}$ ) to be $<15 \%$ to minimize the likelihood of dual labelling. The length of the tether (74 bp, $25 \mathrm{~nm}$ ) was sufficiently long to minimize steric hindrance from the trapped bead on the walking molecule. To test the impact of C-terminal DNA tethers on dynein motility in unloaded conditions, we labelled the distal end of the tether with $\mathrm{Cy} 3$ (Supplementary Fig. 2a) and observed the motility of DNAtagged motors in a single-molecule fluorescence assay (Fig. 2c). Labelled motors remained active and processive, walking at 
a

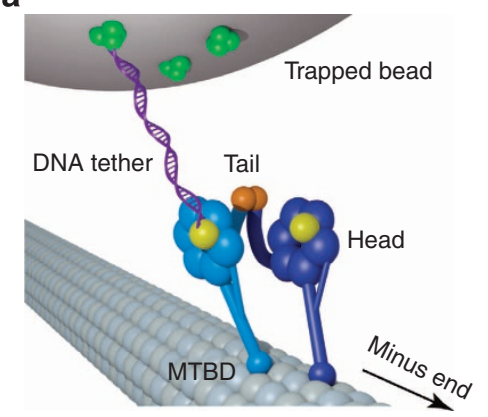

C
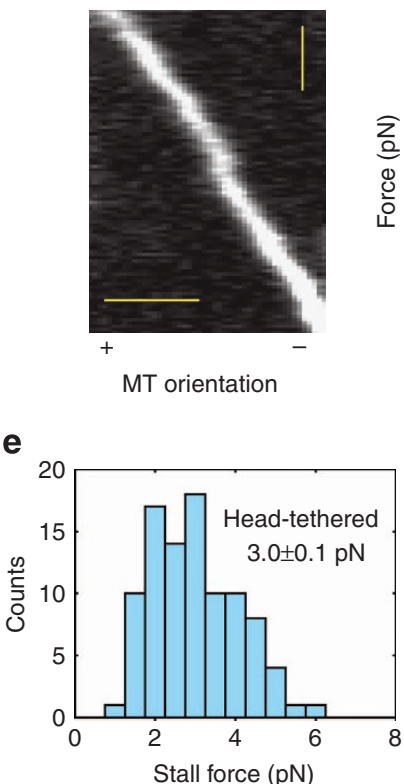

b

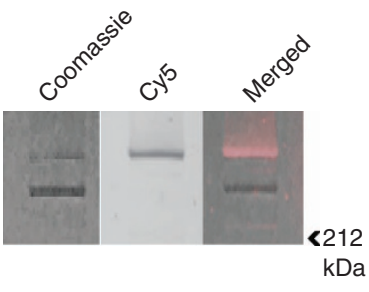

Figure $\mathbf{2}$ | Measuring forces produced by a single dynein head.

(a) Schematic of the head-tethered optical trapping assay for measuring forces produced by a single dynein head. One head of a GST-dimerized motor (GST shown in orange) is labelled with a DNA tether at the C-terminal HT tag (yellow) and tethered to a trapped bead through a biotin-streptavidin (green) linkage. (b) Cy5-DNA-labelled dynein migrates in two distinct bands in a denaturing gel. Fluorescent image identifies the labelled fraction. Labelling efficiency was $31 \%$ in the gel shown here.

(c) Kymograph shows that Cy3-DNA-labelled dynein moves processively towards the MT-minus end. Scale bars: $2 \mu \mathrm{m}$ (horizontal), $10 \mathrm{~s}$ (vertical). (d) Measurement of the stall force of head-tethered dynein. Valid stall events are marked with red ticks. Trap stiffness $\left(k_{\text {trap }}\right)$ is $0.037 \mathrm{pN} \mathrm{nm}^{-1}$. $(\mathbf{e}, \mathbf{f})$ Stall force histograms of head- and tail-tethered dynein motors, respectively (mean \pm s.e.m.; $N=97$ and 123 , respectively).

$98 \pm 9 \mathrm{nms}^{-1}$ (mean \pm s.e.m., $N=73$ ), slightly slower than DNA-free motors tagged with green fluorescent protein (GFP; $117 \pm 7 \mathrm{~nm} \mathrm{~s}^{-1}, N=64$ ). (Supplementary Fig. $2 \mathrm{~b}, \mathrm{c}$ ). These results show that dynein's motility is not significantly hampered by the presence of a DNA tether. Yet, the tether remains relatively rigid $^{18}$ and does not introduce excessive noise into the trap recordings. The distal ends of the DNA tethers were attached to polystyrene beads through a biotin-streptavidin linkage.

Effects of load on stepping and force production of a head. Using a similar geometry, it has been shown that a kinesin- 1 head takes twice the steps' size of the tail; however, its stall force halves relative to that of the tail ${ }^{14}$. This is consistent with the energetics of a kinesin step, which harnesses the hydrolysis energy from one

ATP and produces the same amount of work ${ }^{19}$. Because dynein lacks a regular stepping pattern ${ }^{15,16}$ and possesses multiple ATP-hydrolysis sites, a dynein head may respond differently to external load, which would in turn affect its measured force production. To test this, we compared the stall force of a DNAtethered dynein head with the combined force production through the tail domain (Fig. 2d). Unlike kinesin, the headtethered motor stalls at a force of $3.0 \pm 0.1 \mathrm{pN}$ (mean \pm s.e.m.), similar to the stall force of the tail-tethered motor $(3.1 \pm 0.1 \mathrm{pN}$, mean \pm s.e.m.; two-tailed $t$-test, $P=0.28$; Fig. $2 \mathrm{e}, \mathrm{f})$. These values are in agreement with the tail-tethered force measurements using the same construct (Arne Gennerich, personal communication). Stall forces are not affected by the C-terminal fusion of the HaloTag (HT) domain because a construct with an N-terminal HT fusion stalls at similar forces (Supplementary Fig. 3)

Next, to determine the effect of load on the stepping of a dynein head, we collected and analysed position traces of headand tail-tethered dynein motors under near-stall $(2.5 \mathrm{pN})$ hindering loads in the presence of saturating $(1 \mathrm{mM})$ ATP (Fig. 3a). The size of steps taken by the DNA-tethered head $(12.1 \pm 6.7 \mathrm{~nm}$ backward and $11.0 \pm 5.1 \mathrm{~nm}$ forward steps, mean \pm s.d.) was indistinguishable (two-tailed $t$-test, $P=0.67$ ) from the size of steps measured at the tail $(12.7 \pm 5.7 \mathrm{~nm}$ backward and $12.3 \pm 5.6 \mathrm{~nm}$ forward steps; Fig. 3b). This is consistent with our previous observation that stall forces remain the same between the two geometries because one ATP-driven step is capable of generating a specific amount of work, which is a product of force and step size. Moreover, stepping rates remained similar between the two geometries $\left(10.2 \pm 0.5 \mathrm{~s}^{-1}\right.$ head-tethered, $10.6 \pm 0.5 \mathrm{~s}^{-1}$ tail, mean $\pm 95 \%$ confidence interval; Fig. $\left.3 \mathrm{c}\right)$. This is in contrast to unloaded conditions, in which the head takes nearly twice the centre-of-mass step size and steps less frequently ${ }^{15,17}$. Our findings indicate that the DNA-tethered head decreases its step size to match that of tail-tethered dynein ${ }^{17}$ under load.

Measurement of force generated by dynein's power stroke. The linker swing mechanism is essential for force generation and motility of dynein motors 8,13 . It has been proposed that force is produced in the MT-bound state, as the linker transitions from the primed to unprimed conformation. On the other hand, the linker undergoes a priming transition as it releases from MT, and this was proposed to drive the movement of the stepping head towards the minus end ${ }^{9}$. It remains unclear how much work is performed by each of these conformational changes. To address this question, we measured the stall force and step size of a dynein heterodimer in which one head is rendered catalytically inactive. Force production in one head was abolished by introducing a K/A mutation in the Walker A motif of the AAAl site, preventing ATP from binding ${ }^{20}$. The mutant $\left(\mathrm{AAA}_{\mathrm{K} / \mathrm{A}}\right)$ head was dimerized with a WT head using N-terminal FKBP12 and FRB (FKBPrapamycin binding domain) tags (WT/AAA $1_{\mathrm{K} / \mathrm{A}}$, Fig. $\left.4 \mathrm{a}\right)^{17}$. Constructs dimerized via the FRB-rapamycin-FKBP12 chemistry exhibit similar processivity and stepping properties to GSTdimerized dynein ${ }^{17}$. The stall force of head-tethered FRBFKBP12-dimerized WT/WT dynein is similar $(3.0 \pm 0.1 \mathrm{pN}, t$ test, $P=0.90, N=13$ ) to the GST-dimerized construct, verifying that heterodimerization tags do not hinder the motor's force production.

By attaching the DNA tether to either the WT head or the mutant head, we were able to separately investigate the energetics of the two strokes. Because the mutant $\left(\mathrm{AAA} 1_{\mathrm{K} / \mathrm{A}}\right)$ head remains in an unprimed, tightly bound state and is incapable of undergoing a power stroke ${ }^{12}$, motility is powered solely by the active (WT) head. High-resolution tracking studies of this 
a

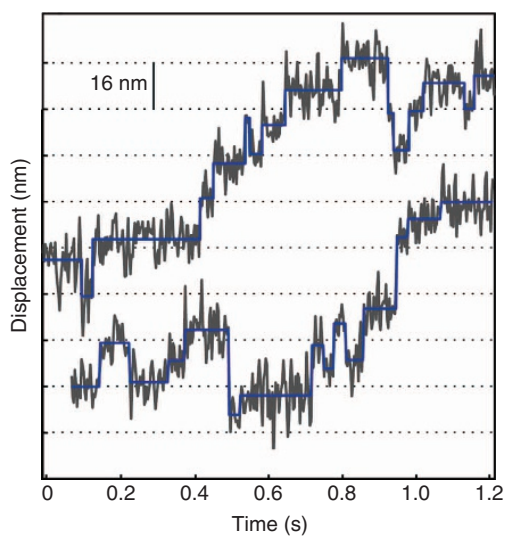

b
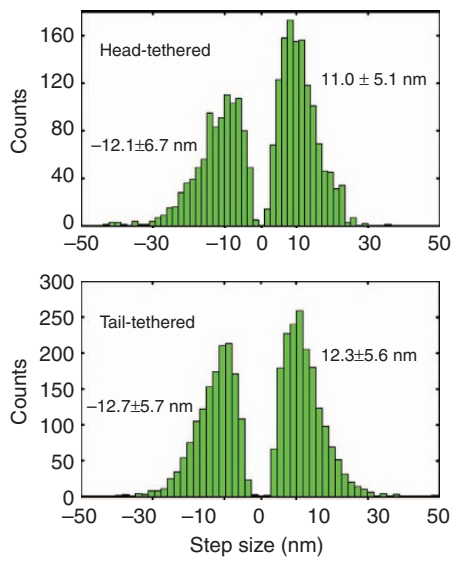

C
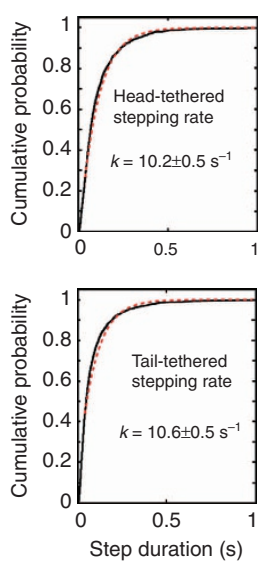

Figure 3 | Observation of steps taken by a dynein head under load. (a) Stepping traces of head-tethered dynein in force-feedback mode under 2.5-pN-hindering load. Position traces (grey lines) are decimated to $330 \mathrm{~Hz}$. The output of the step-fitting algorithm is shown in blue $\left(k_{\text {trap }}=0.025 \mathrm{pN} \mathrm{nm}^{-1}\right)$. (b) Step size distributions of head- and tail-tethered motors under 2.5-pN-hindering load (mean \pm s.d.; $N=2,300$ and 3,293, respectively). (c) Stepping rates $(k)$ of head-tethered and tail-tethered motors under $2.5-\mathrm{pN}$-hindering load, obtained by fitting cumulative probability distributions (solid black lines) to single exponentials (dashed red lines). Reported values are mean $\pm 95 \%$ confidence interval boundaries.

a

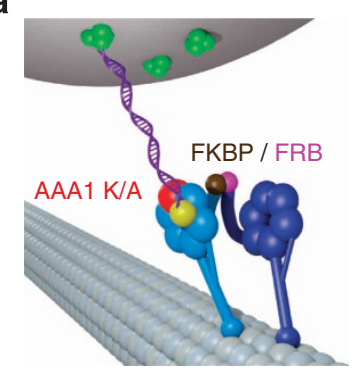

b

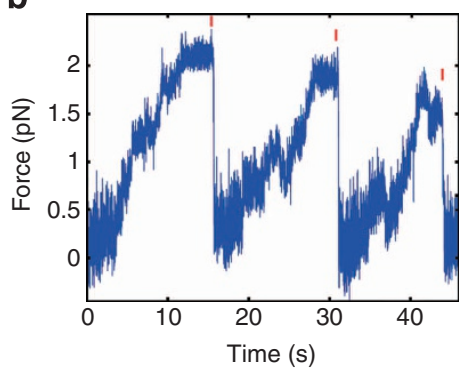

C

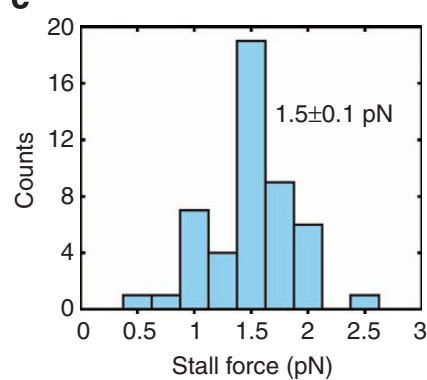

d

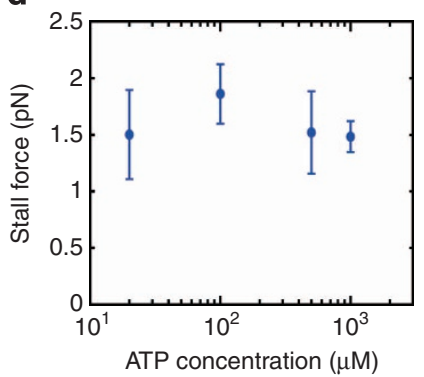

Figure 4 | Behaviour of a mutant head under load. (a) Schematic of the mutant head-tethered optical trapping assay. The DNA tether is attached to an $A A A 1_{K / A}$-mutant head, which cannot bind ATP at the AAA1 site. The mutant head is heterodimerized with a WT head through rapamycininduced FRB-FKBP binding. (b) Stall force measurement of a mutant-head tethered heterodimer at $1 \mathrm{mM}$ ATP $\left(k_{\text {trap }}=0.017 \mathrm{pN} \mathrm{nm}^{-1}\right)$. (c) The mutant head-tethered construct stalls at half the stall force of WT (mean \pm s.d.; $N=48$ ) (d) Stall force of the mutant head-tethered construct is independent of ATP concentration. Error bars represent s.e.m. $N=48-88$ stalls for each data point.

construct showed that WT head mostly remains in the lead and drags the mutant head forward ${ }^{21}$. The force required to pull the mutant head is likely supplied by the power stroke of the WT head and transmitted via linker tension. In contrast, when the tether is directly attached to the sole active head, this head must step forward against load without any assistance provided by its inactive partner. An active dynein head releases from the MT and

moves forward as its linker is primed. It is possible that, during this motion, the MT-bound head serves as an anchor that the stepping head actively pushes away from through the priming stroke of its linker ${ }^{9}$. Therefore, we expect stall force measurements in the WT head-tethered geometry to test whether the priming stroke of the linker produces mechanical work during the stepping of a head.

The WT/AAA $1_{\mathrm{K} / \mathrm{A}}$ heterodimer walks processively at $13.6 \pm 1.2 \mathrm{~nm} \mathrm{~s}^{-1}$. When the DNA tether was attached to the AAA $1_{\mathrm{K} / \mathrm{A}}$ mutant head, the motor stalled at half the stall force of the WT/WT homodimer, $1.5 \pm 0.4 \mathrm{pN}$ (mean \pm s.d., Fig. $4 \mathrm{~b}, \mathrm{c}$ ). Because the DNA-tethered head is catalytically inactive, this assay constitutes a measurement of the minimal amount of force generated by dynein's power stroke. The step size of the mutant head at near-stall $(1.5 \mathrm{pN})$ hindering load was slightly lower than that of the WT head at $2.5-\mathrm{pN}$-hindering load $(9.5 \pm 6.2 \mathrm{~nm}$ for backward steps and $8.5 \pm 5.5 \mathrm{~nm}$ for forward steps, Supplementary Fig. 5a,b), and the stepping rate was $\sim 2.5$ times lower than that of a WT head at $3.6 \pm 0.3 \mathrm{~s}^{-1}$ (Supplementary Fig. 5c). The stall force of a head remained nearly constant across $20 \mu \mathrm{M}$ to $1 \mathrm{mM}$ ATP (Fig. 4d). The lowest tested ATP concentration of $20 \mu \mathrm{M}$ is below the published $K_{\mathrm{M}}$ (ATP) of yeast dynein $^{22}$ and less than half of the $K_{\mathrm{M}}$ (ATP) of the Dyn1 $331 \mathrm{kDa}$ construct used in this study ( $46 \mu \mathrm{M}$, Supplementary Fig. 6). This contrasts with kinesin-1, the stall force of which decreases at limiting ATP concentrations ${ }^{23}$. Our results are consistent with the observation that the stall force of the dynein centre-of-mass does not depend on ATP concentration ${ }^{2}$. Furthermore, because a stalled dynein is in equilibrium between forward and backward steps, our finding suggests that both forward and backward steps are governed by the same ATP-dependent rate and slow down proportionately when ATP is limiting.

Priming stroke does not generate substantial work. To test whether the priming stroke of the linker produces mechanical work as the WT head searches for a new MT-binding site, we attached the DNA tether to the WT head of the heterodimer (Fig. 5a). In contrast with our previous finding that mutant headtethered heterodimers stall at half the stall force of the WT/WT homodimer, we measured the stall force of the live head-tethered construct to be as low as $0.5 \pm 0.1 \mathrm{pN}$ (mean \pm s.d.; Fig. 5b,c). This 
a

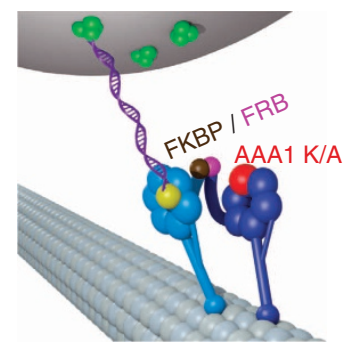

C

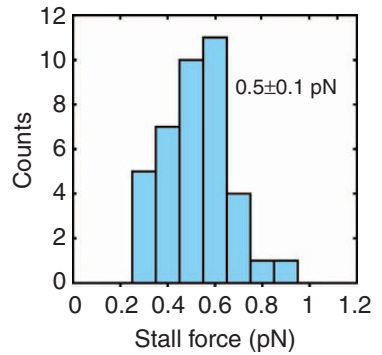

b

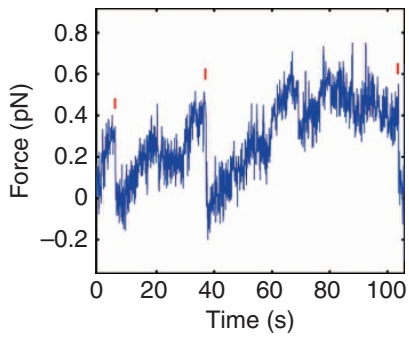

d

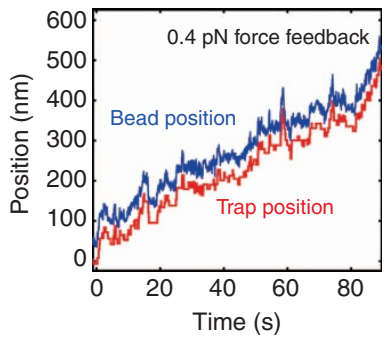

e

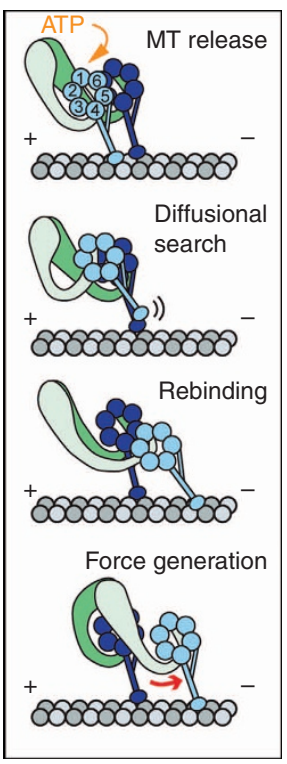

Figure 5 | Characterization of the WT head of a WT/AAA1 $\mathbf{K}_{\mathbf{A}}$ heterodimer under load. (a) Schematic of the WT head-tethered optical trapping assay. The DNA tether is attached to WT head, which is heterodimerized with an AAA1 $/ \mathrm{K}_{\mathrm{A}}$-mutant head. (b) Stall force measurement of the WT-head-tethered heterodimer $\left(k_{\text {trap }}=0.008 \mathrm{pN} \mathrm{nm}^{-1}\right)$. (c) WT-head-tethered heterodimers stall at lower forces $(0.5 \pm 0.1 \mathrm{pN}$, mean $\pm \mathrm{s}$.d.; $N=39)$ than the mutanthead-tethered motors. (d) Despite having a very low stall force, the live-head-tethered construct is able to move processively over long distances against sub-stall $(0.4 \mathrm{pN})$-hindering loads $\left(k_{\text {trap }}=0.008 \mathrm{pN} \mathrm{nm}^{-1}\right)$. (e) Model illustrating dynein force production in the MT-bound state. Upon releasing from the MT, a dynein head undergoes a priming stroke. This component of the step is highly diffusional and can be biased backwards by external load. The force-generating step occurs after the head binds to a new site on the MT lattice.

force corresponds to an energetic bias of $\sim 1.5 \mathrm{k}_{\mathrm{B}} \mathrm{T}$ at room temperature, assuming an average step size of $\sim 12 \mathrm{~nm}^{17}$. Therefore, a single dynein head is only minimally biased to move forward when it is not bound to the MT and is unable to generate appreciable forces in the unbound state. To rule out the possibility that the extremely low forces produced by the WT head-tethered construct may be caused by a loss of processivity upon DNA attachment, we verified that this construct still walks processively for hundreds of nanometres when subjected to substall loads of $0.3-0.4 \mathrm{pN}$ (Fig. $4 \mathrm{~d}$ ). The differences we observe between applying force to the two different heads of the WT/ $\mathrm{AAA} 1_{\mathrm{K} / \mathrm{A}}$ heterodimer can be rationalized by considering the fundamental difference between pulling directly on the 'active' head and pulling on its inactive partner. In the first case, the head must move against the force of the trap at all times, even during the diffusional search for a new binding site after ATP-induced release from the MT. In the second case, the 'active' head is allowed to diffuse freely in the MT-unbound state, while the inactive head remains bound to the track and bears the load exerted by trap. In this case, the active head needs to perform work against the trap in the MT-bound state to pull its inactive partner forward. Our findings show that force required for dynein motility is produced once the stepping head re-binds the MT and the linker returns to its unprimed conformation (Fig. 4e). The priming stroke only generates minimal mechanical work and is insufficient to account for the force generation of a WT dynein dimer.

We next tested the possibilities that interactions between the two AAA + rings or the remaining ATPase-active sites in the mutant head could influence the stall force of WT/AAA $1_{K / A}$. We repeated both WT head-tethered and mutant head-tethered stall force measurements using a construct in which the $\mathrm{AAA} 1_{\mathrm{K} / \mathrm{A}}$ head is replaced with seryl tRNA synthetase (SRS) fused to a dynein MTBD $^{21,24}$ (Supplementary Fig. 7a,b). In this heterodimer, only the 'live' head has a motor domain, while the SRS chimera acts as a true mechanical tether without the possibility of ring stacking or residual motor activity. Despite having only one functional dynein head, this construct walks robustly and is threefold slower but twice as processive as $\mathrm{WT} / \mathrm{WT}^{21}$. We measured the stall force of the SRS-tethered heterodimer to be $1.6 \pm 0.6 \mathrm{pN}$ (mean \pm s.d.), and of the live head-tethered heterodimer to be $0.5 \pm 0.2 \mathrm{pN}$ (mean \pm s.d.) (Supplementary Fig. 7c-f). The results are similar to those of the $\mathrm{WT} / \mathrm{AAAl}_{\mathrm{K} / \mathrm{A}}$ heterodimer (two-tailed $t$-test; SRS-tethered, $P=0.20$; WT head-tethered, $P=0.77$ ). These findings also indicate that tension experienced by the mutant head must be transmitted through the linker, and not through ring-ring interactions.

Force-velocity relationship of a dynein head. To investigate how the overall motility of a dynein dimer is affected by load when one or both heads are able to produce force, we measured the velocities of head-tethered WT/WT and mutant headtethered $\mathrm{WT} / \mathrm{AAA} 1_{\mathrm{K} / \mathrm{A}}$ under varying loads at saturating $(1 \mathrm{mM})$ ATP. The shape of a motor's force-velocity $(\mathrm{F}-\mathrm{V})$ curve is determined by how forward and backward stepping rates are affected by external load ${ }^{23,25}$. Our findings differ from the F-V relationship previously reported for full-length dynein in centreof-mass trapping geometry ${ }^{2}$. The velocities we observe increase continuously as external force transitions from hindering to assisting values. There is no detectable plateau at near-zero forces, suggesting that the principal rate-limiting transition in the step of a dynein head involves substantial movement along the MT axis.

The F-V curve fits well $\left(R^{2}=0.975\right)$ to a simple one-state motor model (Fig. 6a), wherein the movement of a motor is fully described by load-dependent forward and backward stepping rates (see Supplementary Note 1). Interestingly, the best fit 
a

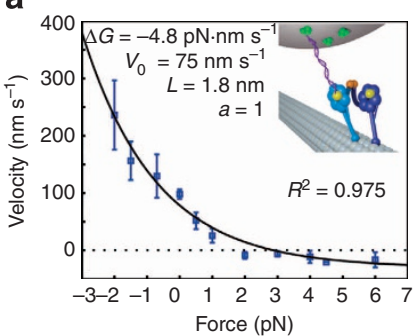

b

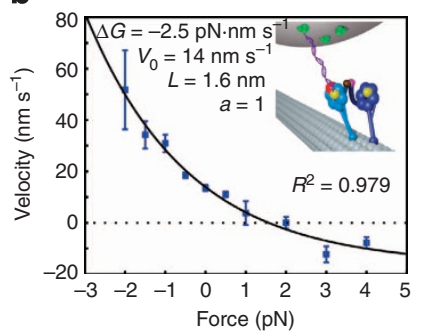

i

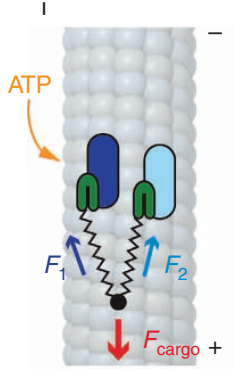

ii

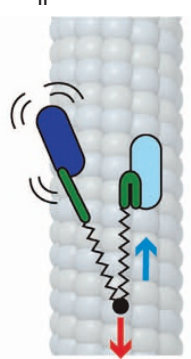

iii

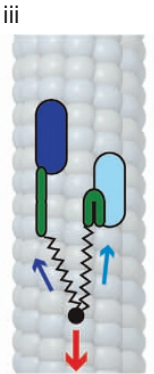

iv

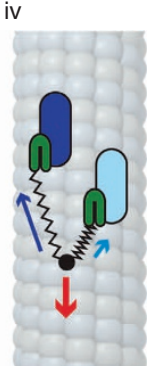

Figure 6 | WT and $\mathbf{A A A 1}_{\mathbf{K} / \mathbf{A}}$ force-velocity relationships. Force-velocity curves for head-tethered WT/WT homodimer (a) and mutant headtethered $A A A 1_{K / A}$ heterodimer (b) at $1 \mathrm{mM}$ ATP are fitted with a one-state model in which both forward and backward stepping rates are loaddependent (black curve). The parameters obtained from the fit are the unloaded velocity $V_{0}$, the characteristic length $L$, the minimum velocity $V_{\text {min, }}$ and the dimensionless parameter $a$ that defines the partitioning of load dependence between the forward and backward stepping rates. Error bars represent s.e.m. $\left(N_{\text {beads }}=4-10\right)$. Velocities at ' $0 \mathrm{pN}$ force' were obtained from single-molecule fluorescence measurements $\left(N_{\text {motor }}>60\right)$.

assigns all load dependence to the forward stepping rate and keeps the backward rate constant across the entire force regime. The load dependence of the forward and backward stepping rates can also be independently estimated from stepping data. Comparing the stepping rates of a DNA-tethered head under 2.5-pN-hindering load to the stepping rate of a dynein head in unloaded conditions ${ }^{15}$, we find that the forward rate decreases from 11.3 to $5.8 \mathrm{~s}^{-1}$, while the backward rate increases from 2.8 to $4.4 \mathrm{~s}^{-1}$. The decrease in forward rate is significantly larger than the increase in backward rate and is responsible for the bulk of the observed dramatic decrease in velocity under load. Meanwhile, the forward step size of the head under load $(11.0 \mathrm{~nm})$ remains very similar to the mean step size of an unloaded head $(10.2 \mathrm{~nm})$, emphasizing the importance of stepping rate rather than step size in velocity measurements. The results are consistent with the finding that dynein monomers release from MT in a force-dependent manner, with assisting (minus-end directed) forces greatly accelerating MT release, and hindering forces having little effect on release rates ${ }^{21}$.

The asymmetric response of a dynein monomer to load could arise from force-dependent inhibition of a particular step in the ATPase cycle. Alternatively, the interaction between the MTBD and the tubulin-binding site may become stronger as force is applied in a particular direction. To distinguish between these possibilities, we repeated $\mathrm{F}-\mathrm{V}$ measurements using the mutant head-tethered WT/AAA $1_{\mathrm{K} / \mathrm{A}}$ construct (Fig. 6b). If the force dependence of velocity of the WT head originated from one of the ATPase steps, such as nucleotide binding or hydrolysis, one would expect a different step to become rate-limiting in the AAA $1_{\mathrm{K} / \mathrm{A}}$ head-tethered construct since this head does not perform an ATP-dependent linker swing ${ }^{12}$. A novel rate-limiting step would in turn result in a different $\mathrm{F}-\mathrm{V}$ relationship. We observed that the mutant head-tethered construct exhibited a concave-up F-V curve, similar to WT/WT. The $\Delta G$ parameter, which represents the effective energetic bias from ATP hydrolysis per step in our simple model, is almost exactly twice as large in WT/WT $(4.8 \mathrm{pNnm})$ as it is in the $\mathrm{AAAl}_{\mathrm{K} / \mathrm{A}}$ head-tethered construct $(2.5 \mathrm{pN} \mathrm{nm})$. This is consistent with our observation that constructs with two active heads are able to pull against twice the stall force of mutant heterodimers. Although WT/AAA $1_{\mathrm{K} / \mathrm{A}}$ is nearly four times slower, the characteristic length $L$, which determines how rapidly the motor's velocity scales with force, remains nearly identical $(1.6 \mathrm{~nm})$ to that of WT/WT $(1.8 \mathrm{~nm})$. The results suggest that the different response of forward and

Figure 7 | Load-sharing model for dynein force production. (i) When dynein is in a two-head bound state, the external load $(F)$ is distributed between the two heads because of the elastic nature of the tail (stretched springs) and linker (bent green rods) regions. Because each panel represents an equilibrium state, the sum of the three forces ( $F_{\text {cargor }}, F_{1}$ and $F_{2}$ ) is always equal to zero. (ii) ATP binding to the AAA1 site of one head triggers its release from the MT and priming of the linker (straight green rod). As this head diffuses to a new binding site, the external load is transferred in its entirety to the DNA-tethered head. (iii) The stepping head rebinds to $M T$, with the linker still in the primed configuration. (iv) The linker returns to its original state via a power stroke, the tail moves forward and the load is redistributed between the heads. Load sharing allows a dynein dimer to work against hindering forces larger than the forces that can be produced by a single head.

backward stepping rates to load arise from the asymmetry of the MT-binding interface. It has been shown that the dynein MTBD behaves as a catch bond under load, as hindering forces slow down its ATP-dependent release from the $\mathrm{MT}^{21}$. The characteristic length arising from our F-V curve may thus correspond to a distance by which the head needs to move in order to release the catch bond of the MTBD.

\section{Discussion}

Characterizing the behaviour of both WT and mutant heads under load allowed us to provide a mechanistic description of the force production by the two heads of a walking dimer. The force is generated mainly in the MT-bound state, consistent with the power stroke model ${ }^{26}$. Our force measurements set a lower bound for the force produced by a power stroke at $1.5 \mathrm{pN}$ (Fig. 4). This force also constitutes a measurement of the maximal force with which one head can pull on its partner head through linker tension. While the priming stroke of the unbound head has been proposed to push against the MT-tethered head and drive motility towards the MT-minus end ${ }^{9}$, we found that it provides less than $1.5 \mathrm{k}_{\mathrm{B}} \mathrm{T}$ of directional bias per step and thus cannot account for the mechanical work performed by dynein over one mechanochemical cycle. However, the priming stroke remains vital to dynein's mechanochemical cycle as it is likely to increase the reach of the diffusional step and prime the linker to enable an effective subsequent power stroke.

We demonstrated that a dynein head is able to generate half the stall force of the dimer. Given dynein's flexibility and lack of strict interhead coordination, the heads in a dynein dimer can be thought of as a pair of mechanically coupled monomers as opposed to a single cyclic machine akin to myosin V or kinesin- 1 . We propose that each of dynein's monomers is capable of independently generating approximately half of the stall force of the dimer, and that each head's force production contributes to the total output of the dimer. We refer to this framework as 'load-sharing' between dynein heads to highlight its similarities with the additive forces observed when a cargo is driven by a low number of processive motors ${ }^{27-29}$. 
Our vision of how load-sharing may be achieved in cytoplasmic dynein is illustrated in Fig. 7. A dynein dimer spends the majority of the time with both heads bound to the MT. Unlike kinesin and myosin, the hindering load is distributed among both heads with their long flexible tails acting as springs (Fig. 7-i). When one head releases from the MT, the entire load is transferred to the bound head (Fig. 7-ii). The bound head functions as an anchor, while the released head performs a diffusional search for a new binding site. During this diffusional search, the tail may briefly move backwards in response to external load; however, we expect this motion to be too rapid to be resolvable, given the $\sim 3$-ms time resolution of our assay. When the stepping head rebinds to MT (Fig. 7-iii) and its linker undergoes a power stroke (Fig. 7-iv), load is redistributed between the heads. The tail of the leading head becomes more stretched, as this head bears a larger fraction of the total load. Since hindering force significantly decreases the rate at which a dynein head releases from the $\mathrm{MT}^{21}$, the leading head now serves as an anchor, while the trailing head becomes more likely to take the subsequent step. Importantly, the work done by the power stroke of each head is stored in the form of linker tension and is applied to bias the subsequent step of the partner head against hindering load. As a result, each step effectively combines the forcegenerating capacities of the two partner heads, allowing a dynein dimer to work against hindering forces higher than the maximal force produced by each head.

A distinguishing feature of dynein's motility under load is that the step size and stepping rate of a DNA-tethered head are the same as the size and rate of steps measured when the load is applied to the tail. This unusual finding can be explained by the large and flexible structure of the dynein dimer and tension-dependent changes in dynein's stepping pattern ${ }^{21}$ (Supplementary Fig. 8).

Our findings further distinguish dynein from other cytoskeletal motors and provide new clues for understanding how it functions in vivo. Unlike kinesin-1, which readily releases from the MT at near-stall loads ${ }^{23}$, the dynein MTBD resists large backwards loads $^{21}$. This may make dynein ideally suited for transportation of large cellular cargos or sliding of MTs under tension. The heads of a dimer produce force independently and they must be inhibited simultaneously by a regulatory protein to stall dynein movement ${ }^{15,30}$. A dimer with a single head inhibited would behave similarly to our $\mathrm{WT} / \mathrm{AAA} 1_{\mathrm{K} / \mathrm{A}}$ heterodimer, slowing down by several fold yet still producing substantial force. Independent force generation can also enable dynein to perform work in unusual environments such as at the ends of MT or while crosslinking two MTs and sliding them relative to each other ${ }^{31}$ and can potentially aid the understanding of collective force production by trimeric outer-arm dyneins ${ }^{32}$. Finally, it has been reported that multiple dyneins combine their efforts more effectively than do multiple kinesins ${ }^{33}$. The load-sharing mechanism inherent to a single dimer may explain dynein's enhanced ability to pull cargos when working in a team. In a system with multiple motors transporting the same cargo, individual motors experiencing a hindering force will tend to remain attached to the track and prevent the cargo from slipping backwards. Meanwhile, motors that are not subjected to a significant hindering force will have a much higher probability of stepping forward, resulting in self-regulating and efficient minusend-directed transport by multiple motors.

\section{Methods}

Preparation of DNA tethers. Double-stranded DNA tethers labelled with HT ligand (Promega) on one end and biotin on the other were prepared as follows. A biotinylated DNA oligo (5'-/biotin/TTCGGTCAATACCCGGCGCAGAGCGC TCAGGCGCGAGGTCAACAGAGGGCGGAGGGTGGGCCAGCGCGACC
CCG-3') was hybridized with an amine-modified DNA oligo (5'-/C6 amino/ GTGTCGGGGTCGCGCTGGCCCACCCTCCGCCCTCTGTTGACCTCGCGC CTGAGCGCTCTGCGCCGGGTATTGAC-3 $\left.3^{\prime}\right)$ in DNA buffer $\left(20 \mathrm{mM} \mathrm{NaHCO}{ }_{3}\right.$, $50 \mathrm{mM} \mathrm{KCl}, 1.25 \mathrm{mM} \mathrm{MgCl}_{2}, \mathrm{pH}=8.4$ ) at $90^{\circ} \mathrm{C}$ for $2 \mathrm{~min}$, followed by incubation at $25^{\circ} \mathrm{C}$ for $40 \mathrm{~min}$. An amine-reactive HT Succinimidyl Ester (O4) ligand (Promega) was then attached to the terminal amine group on the DNA by incubation for $6 \mathrm{~h}$ at room temperature. The reaction was then quenched with $1 \mathrm{mM}$ glycine for $10 \mathrm{~min}$ to remove excess reactive ligand. The resulting tethers were sequentially de-salted into DLB buffer ( $30 \mathrm{mM}$ HEPES, $2 \mathrm{mM} \mathrm{MgCl}_{2}, 1 \mathrm{mM}$ EDTA, $10 \%$ glycerol, pH 7.2) through two G-25 columns (GE Healthcare). De-salted tethers, at $\sim 25 \mu \mathrm{M}$ concentration in DLB, were frozen and stored at $-20^{\circ} \mathrm{C}$. Fluorescently labelled DNA molecules were prepared exactly as described above, except the biotinylated oligo was replaced with a $5^{\prime} \mathrm{Cy} 3$-modified oligo with an identical nucleotide sequence.

Protein purification. S. cerevisiae cultures expressing the dynein constructs used in the study were grown to an optical density (OD)600 of 1.0-1.5. They were then harvested by centrifugation and the pellets were resuspended in $\sim 3 \mathrm{ml}$ of water per litre of starting culture. The resuspended pellets were frozen by dripping into liquid nitrogen and lysed by grinding in a commercial stainless steel coffee grinder (pre-cooled with liquid nitrogen) for $\sim 30 \mathrm{~s}$. Following lysis, the ground powder was thawed in a $37-{ }^{\circ} \mathrm{C}$ water bath concurrently with the addition of 0.2 volumes of $5 \times$ lysis buffer $(150 \mathrm{mM}$ HEPES, $250 \mathrm{mM}$ KAcetate, $10 \mathrm{mM}$ MgAcetate, $5 \mathrm{mM}$ EGTA, $5 \mathrm{mM}$ phenylmethylsulphonyl fluoride (PMSF), $500 \mu \mathrm{M}$ MgATP, $50 \%$ glycerol, pH 7.4). Note that because of their long-term instability in solution, PMSF and MgATP were stored separately and were only added to the stock buffer immediately preceding dynein purification. The resuspended lysis mixture was then centrifuged at $270,000 \mathrm{~g}$ for $45 \mathrm{~min}$. The resulting supernatant was mixed with $200 \mu \mathrm{l}$ of IgG sepharose beads (GE Healthcare Life Sciences) and incubated with rotation at $4{ }^{\circ} \mathrm{C}$ for $1 \mathrm{~h}$. The beads were then washed twice with wash buffer (125 mM KCl, $30 \mathrm{mM}$ HEPES, $50 \mathrm{mM}$ KAcetate, $2 \mathrm{mM}$ MgAcetate, $1 \mathrm{mM}$ EGTA $1 \mathrm{mM}$ PMSF, $100 \mu \mathrm{M}$ MgATP, $10 \%$ glycerol, pH 7.4) and washed once with $10 \mathrm{ml}$ of TEV buffer (150 mM KCl, $10 \mathrm{mM}$ Tris-HCl, $10 \%$ glycerol, $1 \mathrm{mM}$ TCEP, $1 \mathrm{mM}$ PMSF, $100 \mu \mathrm{M}$ ATP, $\mathrm{pH} 8.0$ ). Following the wash, beads were incubated with TEV protease in $500 \mu \mathrm{l}$ of TEV buffer for at $16^{\circ} \mathrm{C}$ for $1 \mathrm{~h}$ with rotation. The dyneincontaining supernatant was then either immediately labelled with DNA tethers or aliquoted and flash-frozen if labelling was not necessary.

Labelling. After TEV cleavage, dynein was mixed with an excess of de-salted tethers (for example, $300 \mu \mathrm{l}$ of $200 \mathrm{nM}$ dynein and $100 \mu \mathrm{l}$ of $25 \mu \mathrm{M}$ tether) and nutated at $4{ }^{\circ} \mathrm{C}$ for $5 \mathrm{~h}$. At the end of the labelling reaction, the microtubule bind and release (MTBR) protocol was used to remove excess DNA and enrich the population of active motors. For MTBR, freshly prepared MTs were suspended in dynein lysis buffer (DLB) supplemented with $10 \mu \mathrm{M}$ taxol (DLB: $30 \mathrm{mM}$ HEPES, $50 \mathrm{mM}$ KAcetate, $2 \mathrm{mM}$ MgAcetate, $1 \mathrm{mM}$ EGTA, $10 \%$ glycerol, $\mathrm{pH}$ 7.4) at a concentration of $\sim 2 \mathrm{mg} \mathrm{ml}^{-1}$, then added to 5 volumes of $\sim 200 \mathrm{nM}$ dynein and incubated for $7 \mathrm{~min}$ at room temperature. The dynein/MT mixture was then layered over $200 \mu \mathrm{l}$ of sucrose cushion (DLB supplemented with $50 \%$ sucrose and $10 \mu \mathrm{M}$ taxol) in an ultracentrifuge tube (Beckman TLA 120.2) and centrifuged at $54,000 \mathrm{~g}$ for $10 \mathrm{~min}$ at $23^{\circ} \mathrm{C}$. The pellet was washed twice with $200 \mu \mathrm{l}$ of wash buffer (DLB supplemented with $1 \mathrm{mM}$ dithiothreitol (DTT) and $10 \mu \mathrm{M}$ taxol) and resuspended in $\sim 200 \mu \mathrm{l}$ of release buffer (DLB supplemented with $300 \mathrm{mM} \mathrm{KCl}$, $2 \mathrm{mM} \mathrm{MgATP}$ and $500 \mu \mathrm{M}$ DTT). The resuspended pellet was allowed to incubate in release buffer for $10 \mathrm{~min}$ at room temperature and then centrifuged at $54,000 \mathrm{~g}$ for $10 \mathrm{~min}$ at $23^{\circ} \mathrm{C}$. The supernatant of this second centrifugation run contained purified DNA-labelled dynein with excess tethers removed. Fractions from MTBR purification were then analysed on a denaturing gel. All dynein constructs used in this study were artificially dimerized with either GST or rapamycin-mediated FKBP-FRB binding (Supplementary Fig. 1). In all, $>96 \%$ of the GST-dimerized constructs were labelled with a single DNA. For tail-tethered dynein experiments, HT-GST-Dyn $1_{331} \mathrm{kD}$ was labelled with a biotinylated HT ligand at the N-terminal HT. The FRB-FKBP pair was used whenever the experiment required heterodimerization between a mutated head and a WT head.

Conjugation of motors to microspeheres. Carboxyl latex beads $(0.9 \mu \mathrm{m}$, Life Technologies) were coated with either streptavidin or custom-made rabbit polyclonal $\alpha$ GFP antibodies (Covance) as follows. The beads were washed four times in activation buffer ( $10 \mathrm{mM}$ MES, $100 \mathrm{mM} \mathrm{NaCl}, \mathrm{pH}$ 6.0) by vortexing, spinning down at $10,000 \mathrm{~g}$ for $3 \mathrm{~min}$ and resuspending. One milligram each of 1-Ethyl-3(3-dimethylaminopropyl)carbodiimide (EDC) and Sulfo-NHS (N-hydroxysulfosuccinimide) crosslinkers (Pierce Biotechnology), dissolved in $100 \mu \mathrm{l}$ of dimethylformamide (DMF) were then added to the beads. The solution was sonicated for $\sim 5 \mathrm{~min}$ and vortexed at low speed until visible clumps disappeared. Afterwards, the beads were washed three times with coupling buffer $(100 \mathrm{mM}$ sodium-phosphate buffer, $\mathrm{pH} 7.4$ ), mixed with streptavidin or antibodies (at $\sim 1 \mathrm{mg}$ of dry streptavidin or antibody per $100 \mu \mathrm{l}$ of $4 \% \mathrm{v} / \mathrm{w}$ bead stock) and reacted by shaking at room temperature for $30 \mathrm{~min}$. The labelled beads were then passivated by adding powdered BSA to a final concentration of $10 \mathrm{mg} \mathrm{ml}^{-1}$ and shaking for $1.5 \mathrm{~h}$ at room temperature. Passivated beads were washed three 
times with $1 \times$ PBS (pH 7.4) and stored at $4{ }^{\circ} \mathrm{C}$ with $0.1 \%$ sodium azide and $0.5 \mathrm{mg} \mathrm{ml}^{-1}$ BSA.

Sample preparation. In FRB-FKBP12 heterodimer experiments, dynein monomers (of which only one was previously labelled with a biotinylated doublestranded DNA tether at the C-terminal HT) were dimerized with $200 \mathrm{nM}$ rapamycin for $10 \mathrm{~min}$ at room temperature before being diluted to the final concentration and mixed with streptavidin-coated beads. In tail-tethered GST homodimer experiments, dynein dimers were diluted to the final concentration and immediately mixed with either $\alpha$ GFP antibody-coated beads (when working with the GFP-GST-Dyn $1_{331 \mathrm{kD}}-\mathrm{HT}$ construct) or streptavidin-coated beads (when working with the biotinylated HT-GST-Dyn $1_{331 \mathrm{kD}}$ construct; Supplementary Fig. 1). The final concentration was determined experimentally for each preparation such that no more than $35 \%$ of beads exhibited any activity when brought in contact with an axoneme, ensuring that $>95 \%$ of observed events can be attributed to the actions of single motors ${ }^{34}$. Dynein was allowed to bind to the beads for $10 \mathrm{~min}$ at $4{ }^{\circ} \mathrm{C}$ before proceeding with sample preparation. The sample chamber was loaded by first flowing Cy5-labelled axonemes in low-salt DLB buffer (30 mM HEPES, $2 \mathrm{mM} \mathrm{MgCl}_{2}, 1 \mathrm{mM}$ EDTA, 10\% glycerol, $\mathrm{pH}$ 7.2), waiting $30 \mathrm{~s}$ for the axonemes to adsorb to the glass surface, and then chasing with a solution of dynein-coated beads in motility buffer (assay DLB as specified above with the addition of $35 \mu \mathrm{g} \mathrm{ml}^{-1}$ protocatechuic acid (PCD), $2.5 \mathrm{mM}$ protocatechuate-3,4dioxygenase (PCA), $10 \mathrm{mM}$ DTT and $1 \mathrm{mg} \mathrm{ml}^{-1}$ casein and $1 \mathrm{mM}$ ATP unless specified otherwise). The ends of the sample chamber were then sealed with nail polish to prevent liquid evaporation.

Optical trapping assay. All trapping experiments were performed on a fully automated optical trap, custom-built in an acoustically isolated and temperaturecontrolled $\left( \pm 0.1{ }^{\circ} \mathrm{C}\right)$ room around the body of a Nikon Ti-E inverted microscope. Dynein-coated beads were trapped with a $2-\mathrm{W} 1,064-\mathrm{nm}$ laser (Coherent), tightly focused in the image plane with a $\times 1001.49$ numeric aperture (N.A.) apochromat oil-immerision objective (Nikon). The trap was steered with a pair of perpendicular acousto-optical deflectors (AA Opto-Electronic), placed in a plane conjugate to the back-focal plane (BFP) of the objective. Power in the trap was controlled throughout the experiment with a half-wave plate on a motorized rotary mount (New Focus). Position of the bead relatively to the centre of the trap was monitored using BFP interferometry, by imaging the BFP of a 1.4 N.A. oil-immersion condenser (Nikon) on a position-sensitive detector (First Sensor Inc.) Raw data from the detector were digitized by an NI 9215 analogue input module (National Instruments), connected to an NI cRIO-9114 reconfigurable chassis (National Instruments). Signals were acquired and saved to disk at either 5 or $20 \mathrm{kHz}$, and position feedback was performed at up to $200 \mathrm{~Hz}$ using the field-programmable gate built into the cRIO-9114 chassis. Detector response was calibrated by rapidly raster-scanning the laser across a trapped bead with the acousto-optical deflectors and fitting the resulting curve to a cubic polynomial (Supplementary Fig. 4c) and trap stiffness was obtained from the Lorentzian fit to the power spectrum of a trapped bead ${ }^{35}$.

The temporal resolution of optical trap data is fundamentally limited by the stiffness of the trap, as Brownian noise dominates the recordings at frequencies higher than the corner frequency of the Lorentzian power spectrum. The trap stiffness values used in this work resulted in corner frequencies of up to $400 \mathrm{~Hz}$, corresponding to a temporal resolution of $\sim 2.5 \mathrm{~ms}$. Owing to the compliance of the dynein motor and the motor-bead linkage, the smallest step size that could be reliably detected by our step-finding algorithm was $\sim 2 \mathrm{~nm}$. The accuracy of position and stiffness calibrations were independently verified by measuring the stall force of kinesin-1 to be $5.6 \pm 0.8 \mathrm{pN}$, in good agreement with previously published values. Axoneme tracks, labelled with $\mathrm{Cy} 5$, were excited with a $633-\mathrm{nm}$ laser (Coherent), visualized with a monochrome camera (The Imaging Source) and brought to the centre of the field of view with a locking XY stage (M-687, Physik Instrumente). The trapped bead was lowered to the surface of the axoneme by moving the trapping objective with a piezo flexure objective scanner (P-721 PIFOC, Physik Instrumente). Only beads that walked for at least $50 \mathrm{~nm}$ against the trap were subjected to force feedback and used for further analysis. To minimize noise, the operator controlled the trap remotely throughout the experiment from an adjacent room through software custom-written in LabVIEW 2012 (National Instruments).

Total internal reflection fluorescence (TIRF) microscopy. Single-molecule motility assays were carried out on a custom-built objective-type TIRF set-up, built around the body of a commercial Nikon Ti-E microscope $\mathrm{e}^{15}$. Individual dyneins labelled with Cy5-tagged DNA tethers were excited with a $30-\mathrm{mW}$ 633-nm laser (Coherent) and imaged with a $\times 1001.49$ N.A. apochromat oil-immersion objective (Nikon) on an iXon EMCCD camera (Andor). Assay preparation was identical to the optical trap sample preparation described in a previous section, except without the addition of latex beads.

Data analysis. Position data were fit to steps using a fully automated, maximum likelihood-based step finding algorithm ${ }^{36}$, implemented in Matlab. Because the algorithm assumes white noise with no autocorrelations, data were downsampled to $\sim 50 \%$ of the corner frequency of the trap before fitting. Force-clamp runs that lasted shorter than $1 \mathrm{~s}$, covered less than $50 \mathrm{~nm}$, or included instantaneous jumps larger than $50 \mathrm{~nm}$, were rejected from the analysis; otherwise the output of the stepfinding algorithm was accepted as is, without any further modifications. Motor velocities were determined by fitting individual runs with a straight line and calculating the slope of the line.

To generate dynein stall force histograms, position data from fixed-trap recordings were downsampled to $500 \mathrm{~Hz}$ for ease of visualization and stall events were manually selected. To qualify as a stall, the position trace had to reach a plateau and remain stationary (with the mean deviations of less than $\pm 10 \mathrm{~nm}$ ) for at least half a second before terminating in a 'rip'. A 'rip', indicating that the motor fully released from the MT, had to constitute a rapid $(<2 \mathrm{~ms})$ jump of at least $50 \mathrm{~nm}$, larger than the maximum step a dynein molecule can be expected to take. For kinesin-1, the definition of stall was the same except the minimum duration of the plateau at the top of the stall was decreased to $0.1 \mathrm{~s}$, to account for kinesin's faster stepping rate and shorter near-stall dwells.

$\mathrm{F}-\mathrm{V}$ curves were fit to the velocity expression for a one-state motor model ${ }^{25}$, $V(F)=V_{+} e^{-a F L / k T}\left(1-e^{(\Delta G+F L) / k T}\right)$, where $F$ is the external force, $V_{+}$is the unloaded velocity because of forward stepping only, $\Delta G$ is the energetic bias provided by ATP hydrolysis, $L$ is a characteristic distance and $a$ is a dimensionless parameter that defines the partitioning of load dependence between the forward and backward stepping rates. The net unloaded velocity $V_{0}$ can be expressed as $V_{0}=V_{+}\left(1-e^{\Delta G / k T}\right)$. See Supplementary Note 1 for detailed description of the model.

\section{References}

1. Mallik, R., Carter, B. C., Lex, S. A., King, S. J. \& Gross, S. P. Cytoplasmic dynein functions as a gear in response to load. Nature 427, 649-652 (2004).

2. Gennerich, A., Carter, A. P., Reck-Peterson, S. L. \& Vale, R. D. Force-induced bidirectional stepping of cytoplasmic dynein. Cell 131, 952-965 (2007).

3. Roberts, A. J., Kon, T., Knight, P. J., Sutoh, K. \& Burgess, S. A. Functions and mechanics of dynein motor proteins. Nat. Rev. Mol. Cell Biol. 14, 713-726 (2013).

4. Kardon, J. R. \& Vale, R. D. Regulators of the cytoplasmic dynein motor. Nat. Rev. Mol. Cell Biol. 10, 854-865 (2009).

5. Carter, A. P., Cho, C., Jin, L. \& Vale, R. D. Crystal structure of the dynein motor domain. Science 331, 1159-1165 (2011).

6. Kon, T., Sutoh, K. \& Kurisu, G. X-ray structure of a functional full-length dynein motor domain. Nat. Struct. Mol. Biol. 18, 638-642 (2011).

7. Höök, P. \& Vallee, R. B. The dynein family at a glance. J. Cell Sci. 119, 4369-4371 (2006).

8. Imamula, K., Kon, T., Ohkura, R. \& Sutoh, K. The coordination of cyclic microtubule association/dissociation and tail swing of cytoplasmic dynein. Proc. Natl Acad. Sci. USA 104, 16134-16139 (2007).

9. Roberts, A. J. et al. AAA + Ring and linker swing mechanism in the dynein motor. Cell 136, 485-495 (2009).

10. Kon, T. et al. The $2.8 \AA$ crystal structure of the dynein motor domain. Nature 484, 345-350 (2012).

11. Schmidt, H., Gleave, E. S. \& Carter, A. P. Insights into dynein motor domain function from a 3.3-Å crystal structure. Nat. Struct. Mol. Biol. 19, 492-497 (2012).

12. Kon, T., Mogami, T., Ohkura, R., Nishiura, M. \& Sutoh, K. ATP hydrolysis cycle-dependent tail motions in cytoplasmic dynein. Nat. Struct. Mol. Biol. 12 513-519 (2005).

13. Roberts, A. J. et al. ATP-driven remodeling of the linker domain in the Dynein motor. Structure 20, 1670-1680 (2012).

14. Guydosh, N. R. \& Block, S. M. Direct observation of the binding state of the kinesin head to the microtubule. Nature 461, 125-128 (2009).

15. DeWitt, M. A., Chang, A. Y., Combs, P. A. \& Yildiz, A. Cytoplasmic dynein moves through uncoordinated stepping of the AAA + ring domains. Science 335, 221-225 (2012).

16. Qiu, W. et al. Dynein achieves processive motion using both stochastic and coordinated stepping. Nat. Struct. Mol. Biol. 19, 193-200 (2012).

17. Reck-Peterson, S. L. et al. Single-molecule analysis of dynein processivity and stepping behavior. Cell 126, 335-348 (2006).

18. Lu, Y., Weers, B. \& Stellwagen, N. C. DNA persistence length revisited. Biopolymers 61, 261-275 (2002).

19. Coy, D., Wagenbach, M. \& Howard, J. Kinesin takes one 8-nm step for each ATP that it hydrolyzes. J. Biol. Chem. 274, 3667-3671 (1999).

20. Kon, T., Nishiura, M., Ohkura, R., Toyoshima, Y. Y. \& Sutoh, K. Distinct functions of nucleotide-binding/hydrolysis sites in the four AAA modules of cytoplasmic dynein. Biochemistry 43, 11266-11274 (2004).

21. Cleary, F. B. et al. Tension on the linker gates the ATP-dependent release of dynein from microtubules. Nat. Commun. 5, 4587 (2014)

22. Cho, C., Reck-Peterson, S. L. \& Vale, R. D. Regulatory ATPase sites of cytoplasmic dynein affect processivity and force generation. J. Biol. Chem. 283, 25839-25845 (2008)

23. Visscher, K., Schnitzer, M. J. \& Block, S. M. Single kinesin molecules studied with a molecular force clamp. Nature 400, 184-189 (1999). 
24. Gibbons, I. R. et al. The affinity of the dynein microtubule-binding domain is modulated by the conformation of its coiled-coil stalk. J. Biol. Chem. 280, 23960-23965 (2005)

25. Thomas, N., Imafuku, Y. \& Tawada, K. Molecular motors: thermodynamics and the random walk. Proc. R. Soc. B 268, 2113-2122 (2001).

26. Burgess, S. a, Walker, M. L., Sakakibara, H., Knight, P. J. \& Oiwa, K. Dynein structure and power stroke. Nature 421, 715-718 (2003).

27. Hendricks, A. G., Holzbaur, E. L. F. \& Goldman, Y. E. Force measurements on cargoes in living cells reveal collective dynamics of microtubule motors. Proc. Natl Acad. Sci. USA 2012, 1-6 (2012).

28. Rai, A. K., Rai, A., Ramaiya, A. J., Jha, R. \& Mallik, R. Molecular adaptations allow dynein to generate large collective forces inside cells. Cell 152, 172-182 (2013).

29. Kunwar, A. \& Mogilner, A. Robust transport by multiple motors with nonlinear force-velocity relations and stochastic load sharing. Phys. Biol. 7, 16012 (2010).

30. Huang, J., Roberts, A. J., Leschziner, A. E. \& Reck-Peterson, S. L. Lis1 Acts as a "Clutch" between the ATPase and microtubule-binding domains of the Dynein motor. Cell 150, 975-986 (2012).

31. Tanenbaum, M. E., Vale, R. D. \& McKenney, R. J. Cytoplasmic dynein crosslinks and slides anti-parallel microtubules using its two motor domains. Elife 2, e00943 (2013).

32. Hirakawa, E., Higuchi, H. \& Toyoshima, Y. Y. Processive movement of single $22 S$ dynein molecules occurs only at low ATP concentrations. Proc. Natl Acad. Sci. USA 97, 2533-2537 (2000).

33. Derr, N. D. et al. Tug-of-War in motor protein ensembles revealed with a programmable DNA origami scaffold. Science 338, 662-665 (2012).

34. Svoboda, K. \& Block, S. M. Force and velocity measured for single kinesin molecules. Cell 77, 773-784 (1994).
35. Neuman, K. C. \& Block, S. M. Optical trapping. Rev. Sci. Instrum. 75, 2787-2809 (2004).

36. Kalafut, B. \& Visscher, K. An objective, model-independent method for detection of non-uniform steps in noisy signals. Comput. Phys. Commun. 179, 716-723 (2008).

\section{Acknowledgements}

We are grateful to F.B. Cleary, M.A. Dewitt, J. Bandaria and C.A. Cypranowska for critical reading of this manuscript. This work was supported by NIH (GM094522 (A.Y.)), NSF CAREER Award (MCB-1055017 (A.Y.)) and NSF Graduate Research Fellowship (DGE 1106400 (V.B.)).

\section{Author contributions}

V.B. and A.Y. designed experiments; V.B. performed the biochemical experiments; V.B., N.L.H. and A.C. performed optical trapping assays, V.B., N.L.H. and A.C. analysed the data and V.B. and A.Y. wrote the manuscript

\section{Additional information}

Supplementary Information accompanies this paper at http://www.nature.com/ naturecommunications

Competing financial interests: The authors declare no competing financial interests.

Reprints and permission information is available online at http://npg.nature.com/ reprintsandpermissions/

How to cite this article: Belyy, V. et al. Cytoplasmic dynein transports cargos via loadsharing between the heads. Nat. Commun. 5:5544 doi: 10.1038/ncomms6544 (2014). 